\title{
La construction des valeurs en éducation relative à l'environnement
}

\section{Maryse Clary}

\section{(2) OpenEdition \\ Journals}

Édition électronique

URL : http://journals.openedition.org/ere/1960

DOI : $10.4000 /$ ere. 1960

ISSN : 2561-2271

Éditeur

Centr'ERE

Référence électronique

Maryse Clary, «La construction des valeurs en éducation relative à l'environnement », Éducation relative à l'environnement [En ligne], Volume 8 | 2009, mis en ligne le 20 décembre 2009, consulté le 21 février 2020. URL : http://journals.openedition.org/ere/1960 ; DOI : 10.4000/ere.1960 


\title{
La construction des valeurs en éducation relative à l'environnement
}

\author{
Maryse Clary
}

1 Selon une idée fort répandue, nous traversons aujourd'hui une crise des valeurs. Loin de nous la prétention de nous interroger sur la crise ou le devenir des valeurs dans un monde de plus en plus globalisé. Cependant, puisque crise il y a, est-ce à dire que nous allons vers un monde sans éthique ? Nous ne le pensons pas. Il y a toujours des valeurs. Il n'y a même jamais eu, dans l'histoire de l'humanité, autant de valeurs en présence. La pluralité des cultures à laquelle nous sommes actuellement confrontés ne révèle-t-elle pas un pluralisme des valeurs si bien que le problème deviendrait « comment s'orienter parmi toutes ces valeurs? » Paul Valéry avait déjà remarqué que notre conception des valeurs morales ou esthétiques tendait à se rapprocher, dans un monde dominé par la spéculation, du modèle de la valeur boursière. Il n'y a plus d'étalon fixe des valeurs : nous vivons dans un monde dominé par l'éphémère, la mode, le caprice subjectif. Comment alors la question centrale de l'éducation peut-elle trouver sa place dans un monde fluctuant, marqué par la valorisation d'images éphémères? Dans un monde de plus en plus dominé par les mobiles d'intérêt économique et les valeurs matérialistes de consommation, de satisfaction à court terme émergent de nouvelles valeurs à la fois cognitives et prospectives, moins héritées qu'inventées.

2 Parce qu'elles confèrent un sens profond à nos actions et à notre vie, la construction des valeurs revêt une importance fondamentale en éducation relative à l'environnement (ErE). Lorsqu'on parle d'ErE, ce qui apparaît en premier, c'est le terme éducation. L'éducation a un sens plus large que l'instruction. Elle vise avant tout une construction des valeurs morales alors que l'instruction vise à la formation intellectuelle de l'individu. Éduquer, nous dit le Petit Larousse, c'est « développer les capacités intellectuelles et physiques et le sens moral de quelqu'un». Étymologiquement, éduquer signifie conduire hors de. Dans Voici le temps du monde fini, 
Albert Jacquard (1991) nous amène à réfléchir sur la dérive qu'a subie le terme éducation :

Educere, conduire un enfant hors de lui-même, l'inciter à s'auto-construire, lui en donner les moyens, n'est-ce pas le rôle premier de toute société ? Peu à peu, on a transformé ce rôle en un apport de savoir, de nourriture. L'enfant est gavé pour qu'il en sache plus que les autres, qu'il soit compétitif, qu'il devienne un gagnant, qu'il fasse des perdants. Educere (conduire hors de) s'est dégradé en educare (nourrir). (Jacquard, 1991)

Il y a donc toute une réflexion à mener sur les finalités de l'éducation dans la perspective d'un développement viable pour tous. L'éducation est en quelque sorte tenue de fournir les cartes d'un monde complexe et la boussole permettant d'y naviguer. Une réponse purement quantitative en terme d'acquisition d'un stock de connaissances n'est ni possible ni appropriée. Il faut que chaque individu soit en mesure de saisir et d'exploiter d'un bout à l'autre de son existence toutes les occasions de mettre à jour, d'approfondir et d'enrichir ses connaissances ainsi que de s'adapter à un monde changeant. L'éducation relative à l'environnement constitue un ensemble complexe recouvrant tout à la fois l'adhésion à des valeurs, l'acquisition de connaissances et l'apprentissage de pratiques de participation à la vie publique. Elle ne peut donc être considérée comme neutre du point de vue idéologique. Elle interroge la conscience de l'élève. Pour sauvegarder l'indépendance de celle-ci, l'éducation, dans son ensemble, doit forger aussi la capacité critique qui permet une pensée libre et une action autonome.

\section{Initier à la complexité comme un problème éthique}

4 Les problèmes liés à l'environnement et au développement durable sont caractérisés par leur complexité. Cette complexité, il faut la connaître et la comprendre pour ne pas tomber dans la simplification qui est la porte ouverte à toutes les déviances et qui est un acte irresponsable de la part de ceux qui comprennent les problèmes. Or, il y a une inadéquation de plus en plus grande entre des situations réelles ou des problèmes de plus en plus transversaux et nos savoirs cloisonnés, compartimentés. Une tradition de pensée bien enracinée dans notre culture et qui forme les esprits dès l'école élémentaire nous amène à connaitre le monde par des idées claires et distinctes et à disjoindre ce qui est lié, à unifier ce qui est multiple. La mise du savoir en disciplines a amené de l'ordre et de l'organisation dans l'enseignement, mais elle a aussi entraîné une perte de sens, la standardisation des savoirs ayant souvent donné l'illusion de la transférabilité. La connaissance d'informations isolées est insuffisante, ce n'est que dans le contexte qu'elles prennent sens.

De ce fait, l'ErE se trouve en rupture par rapport aux savoirs traditionnels et Edgar Morin (1992) va jusqu'à parler de pensée écologisée. En effet, ainsi que le précise cet auteur, « le problème crucial de notre temps est celui de la nécessité d'une pensée apte à relever le défi de la complexité du réel, c'est-à-dire de saisir les liaisons, interactions et implications mutuelles » (Morin, 2007). Tout problème d'environnement relève de plusieurs champs à la fois qui s'entrecroisent, interagissent les uns les autres, créant des phénomènes nouveaux parfois irréversibles. C'est ce processus de complexité que doit saisir l'éducation relative à l'environnement. "Il ne s'agit pas de reprendre l'ambition de la pensée simple qui était de contrôler le réel, mais de s'exercer à une pensée capable de traiter avec le réel, de dialoguer, de négocier » (Morin, 1990, avant- 
propos). La complexité, "un des maitres mots du discours scientifique actuel» (Jacquard, 1991, p. 84), caractérise une structure composée de nombreux éléments, appartenant à de nombreuses catégories, ayant chacun des caractéristiques, développant entre eux de multiples interactions non linéaires et échangeant avec un environnement externe. Les unités complexes sont multidimensionnelles.

6 Si les savoirs disciplinaires sont organisés autour de questions structurées par des présupposés théoriques, l'ErE se structure autour de problèmes concrets à résoudre, en fonction du contexte et de la manière dont on veut agir, on vise des situations particulières. Ainsi que l'analyse Claude Bastien (1992), « l'évolution cognitive ne va pas vers la mise en place de connaissances de plus en plus abstraites, mais, à l'inverse, vers leur mise en contexte" et de préciser «la contextualisation est une condition essentielle de l'efficacité ». Cependant, la réalité n'est pas lisible de toute évidence. Les idées et les théories ne reflètent pas, mais interprètent la réalité, ce qu'elles peuvent faire de façon tout à fait erronée. Il importe donc de comprendre l'incertitude du réel, savoir qu'il y a du possible encore invisible dans le réel. Les représentations que se donnent les scientifiques d'une situation-problème, même si elles utilisent des résultats disciplinaires, ne sont pertinentes que dans la mesure où elles sont organisées en fonction de ce que l'on veut faire. On construit alors une représentation théorique appropriée pour pouvoir communiquer et décider dans un contexte précis et en fonction d'un projet particulier. Gérard Fourez (1998) évoque la construction d'un îlot de rationalité et nous renvoie à 2 métaphores : d'une part, « un îlot émergeant dans un océan d'ignorance » et, d'autre part, "celle de rationalité qui implique la possibilité d'une discussion parce qu'on s'est mis suffisamment d'accord sur ce dont on parle ».

7 Le savoir devient un lieu de complémentarité entre les savoirs disciplinaires, les savoirs interdisciplinaires, les savoirs de terrain et ceux qui émergent de la vie quotidienne. La notion de négociation devient alors fondamentale. La méthode générale s'apparente à la démarche de résolution de problème orientée vers la compréhension de la situation en vue d'une prise de décision éclairée. L'éducation doit donc favoriser l'aptitude naturelle de l'esprit à se poser des questions, laisser s'exercer librement la curiosité de l'enfant, de l'adolescent, la stimuler alors que trop souvent l'éducation traditionnelle l'éteint. Elle doit amener les élèves à se référer au complexe, au contexte, de façon multidimensionnelle et dans une conception globale.

\section{Comprendre le monde, comprendre l'autre}

8 Nous devons constater que, selon le mot de Paul Valéry, la planète est « finie », et que cette finitude implique la solidarité des humains. Cependant, comme l'analyse Albert Jacquard (2000, p. 77), «ce mot solidarité n'évoque pas ici un bon sentiment, mais la réalité d'une interdépendance. Les Hommes n'ont pas le choix d'être ou non solidaires ; ils le sont par nécessité, comme les passagers uniques d'un bateau ». Et de prendre l'exemple de l'effet de serre. Aussi, aider à transformer une interdépendance de fait en une solidarité voulue correspond à une des tâches essentielles de l'éducation. Elle doit, à cette fin, mettre chaque individu en mesure de se comprendre lui-même et de comprendre l'autre à travers une meilleure connaissance du monde. Pour que chacun puisse saisir la complexité grandissante des phénomènes mondiaux et dominer le sentiment d'incertitude qu'elle suscite, il lui faut d'abord acquérir un ensemble de connaissances, puis apprendre à relativiser les faits et à faire preuve de sens critique 
face au flux des informations. L'éducation manifeste ici, plus que jamais, son caractère irremplaçable dans la formation du jugement. Elle favorise une compréhension véritable des événements, au-delà de la vision simplificatrice et par là même déformée qu'en donnent les médias. La compréhension de ce monde passe par celle des rapports qui unissent l'être humain à son environnement.

9 L'exigence d'une solidarité planétaire suppose que soient dépassées les tendances au repli identitaire au profit d'une compréhension des autres fondée sur le respect de la diversité. La responsabilité de l'éducation dans cette démarche est primordiale car affirmer sa différence peut constituer une démarche positive, mais mal comprise, cette revendication contribue à rendre difficiles la rencontre et le dialogue avec l'autre. "L'éducation doit dès lors s'attacher à la fois à rendre l'individu conscient de ses racines, afin qu'il puisse disposer de repères permettant de se situer dans le monde, et à lui apprendre le respect des autres cultures »(Delors, 1996, p. 48). La connaissance des autres cultures conduit à une double prise de conscience : celle de la singularité de sa propre culture, mais aussi celle d'un patrimoine commun à l'ensemble de l'humanité. L'éducation a pour mission d'enseigner à la fois la diversité de l'espèce humaine et la conscience des similitudes et de l'interdépendance entre tous les êtres humains de la planète. Or, la diversité culturelle de l'humanité est aujourd'hui en péril. À cet égard, on peut établir un parallèle entre la diversité biologique et la diversité culturelle, considérées comme deux aspects d'un même phénomène.

L'individu se construit dans l'interaction dynamique identité-altérité. La découverte de l'autre passe nécessairement par la connaissance de soi et, pour donner à l'enfant et à l'adolescent une vision juste du monde, l'éducation doit d'abord lui faire découvrir qui il est. C'est alors seulement qu'il pourra se mettre à la place des autres pour comprendre leurs réactions. Mais, comprendre les autres permet de mieux se connaître soi-même. Toute forme d'identité est complexe, chaque individu se définissant par rapport à l'autre, aux autres, à plusieurs groupes d'appartenance. La découverte de la multiplicité de ces appartenances conduit à la recherche de valeurs communes propres à fonder une solidarité intellectuelle et morale. Apprendre à vivre ensemble en développant la compréhension d'autrui et de son histoire, de ses traditions et valeurs spirituelles, l'objectif étant d'encourager les gens à réaliser des projets à plusieurs et à gérer les conflits de façon intelligente et pacifique. L'éducation à l'environnement est aussi une éducation à la diversité bioculturelle, à la convergence entre nature et cultures.

\section{Une éthique de la responsabilité}

L'éducation doit contribuer au développement total de chaque individu et conduire chacun à être en mesure de se constituer une pensée autonome et critique, être capable de se forger son propre jugement. Le but essentiel de l'éducation relative à l'environnement et à la viabilité est de promouvoir une nouvelle citoyenneté contribuant à doter les jeunes d'attitudes et de comportements visant à respecter, protéger et finalement bien gérer l'environnement, à avoir à l'égard des autres une attitude de compréhension, de tolérance et d'entraide, et surtout, se référer à la notion de responsabilité et d'engagement, au service d'une gestion raisonnée et raisonnable du territoire, au service d'une protection intelligente de notre cadre de vie, au service d'un fonctionnement harmonieux de nos rapports sociaux. Quel terrain d'apprentissage 
privilégier ? Celui sur lequel on pourra agir au quotidien, à savoir son propre territoire. Seules la compréhension de son propre territoire, la maîtrise de son espace, la mémoire qui s'y rattache permettent de s'insérer dans la société et d'adhérer à des choix plus vastes mettant en jeu la planète. Il s'agit de s'approprier l'espace et la mémoire du lieu non pas dans un sens conservatoire, mais pour mieux maitriser son futur, être un acteur responsable dans le changement.

Cette éthique de la responsabilité s'avère d'autant plus indispensable que le monde dans lequel nous vivons connaît un changement majeur : les Hommes ont désormais les moyens techniques d'altérer et de détruire de manière irréversible leur environnement. "Les Hommes ont commencé à mettre en danger la planète sur laquelle ils vivent, la seule planète qui, précisément, a rendu possible le développement des formes de vie, au sein desquelles est apparue et s'est affirmée la vie humaine » (Béaud et al., 1993). Nous portons atteinte au vivant en prélevant et en gaspillant sans discernement les ressources, en déstabilisant l'environnement, en bouleversant l'équilibre naturel, et l'on sait maintenant que le climat de la planète est en train de changer à cause des activités humaines. Eric Cobast (2005), dans un écrit intitulé "L'insoutenable responsabilité des Hommes" analyse comment les Anciens, par le moyen de la technique, «achevaient » la Nature dans le sens où ils révélaient tout ce qu'elle pouvait donner et comment ce sens s'est inversé si bien que la Nature, de fin est devenue un moyen.

Et la dimension éthique s'avère fondamentale dans la mesure où, nous dit-il :

Instrumentaliser de la sorte la Nature, c'est aussi prendre l'habitude de faire de l'Homme, un matériau, une matière première. On donnerait aujourd'hui plus volontiers comme exemple de cette dérive, trahie par le langage, la douloureuse expression de "ressources humaines" que d'aucuns dans les entreprises ont la charge de gérer. (Cobast, 2005)

14 Aussi, pouvons-nous dire que ce qui est en jeu, c'est l'Homme lui-même, ce sont les relations entre les Hommes et, avec l'avenir de l'Homme et de la Terre, c'est l'humanisme.

Nous nous réfèrerons à Edgar Morin (1999, p. 47) pour dire avec lui que «la connaissance est une navigation dans un océan d'incertitudes à travers des archipels de certitudes». Cette nouvelle approche scientifique, appuyée sur un principe d'incertitude, tout particulièrement en ce qui concerne les questions d'environnement, a pour corollaire une éthique de la responsabilité. Nous devons apprendre, comme le propose Ravetz (1992) «à nous servir de l'ignorance comme nous nous servons de la connaissance ", l'incertitude devenant l'autre versant de la connaissance. L'insuffisance de preuves pouvant engendrer aussi bien l'action que l'inaction, Hans Jonas (1990, cité par Theys et Kalaora, 1992, p. 33) insiste, lui aussi, sur le caractère complémentaire du savoir et de l'ignorance et dit-il «face à des risques de type planétaire, il convient de traiter le doute comme une certitude possible et donc un élément fondamentalement positif de la décision ». Ce qui est nouveau, c'est l'irréversibilité qui menace, aussi reste-t-il à prendre, avant qu'il ne soit trop tard, les mesures correctives qui s'imposent et on pourrait, à ce propos, parler d'" éthique du temps", ce qui correspond à l'impératif moral d'agir avant que ne soit atteint le point de non retour. L'humanité détient donc à la fois les clés du pouvoir et de la responsabilité, non seulement vis-à-vis des gens vivant actuellement sur la Terre, mais aussi à l'égard des générations futures : «ce qui est véritablement en jeu, ce sont les droits de l'Homme, pas seulement de 
l'Homme d'aujourd'hui, mais aussi de ses enfants et ses petits-enfants» (CMED, 1988). On pourrait évoquer la nécessité d'un lien éthique entre le passé, le présent et l'avenir.

François Ewald (1992) va jusqu'à évoquer une démocratie de l'expertise: «l'environnement désigne un espace de débat sur les valeurs, où se trouve posée la question de la valeur des valeurs qui président à nos sociétés. L'institution politique de l'environnement est l'institution d'un tel espace comme espace du débat démocratique ». Ce type de débat engage de nouvelles pratiques démocratiques: apprendre à gérer les conflits, refuser les réponses définitives et les certitudes, ainsi que le développement d'une vision moins mécaniste de l'Homme. Préoccupations éthiques et humanistes se rejoignent dans une approche surmontant la séparation entre connaissances et valeurs. Elles mettent en avant la notion d'engagement, engagement au service d'une gestion raisonnée et raisonnable du territoire, au service d'une protection intelligente de notre cadre de vie, au service d'un fonctionnement harmonieux de nos rapports sociaux. L'ErE apparaît comme l'une des formes majeures de la formation civique, elle contribue non seulement à faire connaître les mécanismes biophysiques, économiques et sociaux qui régissent les relations de l'Homme et de son milieu, mais aussi à développer une morale de la responsabilité, seule capable de maintenir le fragile équilibre terrestre et de renouveler le pacte fondamental unissant l'Homme à la nature et les Hommes entre eux.

\section{En conclusion}

$17 \mathrm{Au}$ plan comportemental, il s'agit de faire cesser l'indifférence des gens envers la nature, de leur permettre de s'approprier l'environnement, leur environnement, dans l'espace et dans le temps, à nouer une relation directe au monde, c'est-à-dire établir sa propre identité. Cette conscience identitaire permet de construire une citoyenneté reposant sur la responsabilité et la solidarité, valeurs toutes deux associées à la liberté, une liberté pour laquelle l'autonomie est laissée à l'individu et au groupe. À chacun de s'informer et de participer en individu responsable, encore faut-il que chacun ait la possibilité de participer activement à la prise de décision concernant l'environnement dans lequel il vit.

18 L'ErE doit amener à la compréhension des systèmes de valeurs qui la sous-tendent et à la recherche de valeurs mieux adaptées à un développement durable. Ainsi, intégrer la responsabilité envers les générations futures dans les décisions d'aujourd'hui est un choix éthique. Développer une citoyenneté de proximité, bien sûr, comme forme privilégiée d'intervention sur le monde vécu de chaque collectivité, mais aussi une citoyenneté planétaire. Comment élargir la question des droits de l'Homme à celle, non moins cruciale, de ses devoirs à l'égard des générations futures et également à l'égard des autres espèces et de la nature?

19 La problématique de l'environnement est apparue quand les sociétés ont ressenti leur propre développement comme une menace pour la survie de l'espèce humaine. Dans cette mesure, la finalité de la science est devenue politique. Science de la complexité, elle met en lumière le caractère complémentaire et non contradictoire des sciences expérimentales et des sciences sociales. Dans la mesure où le savoir sera nécessaire pour le changement, le lien entre l'éthique et la science pourra être la clé de résolution de bien des problèmes qui concernent notre avenir. 


\section{BIBLIOGRAPHIE}

Bastien, C. (1992). Le décalage entre logique et connaissance. Le Courrier du CNRS, 79, 38.

Béaud, M., Béaud, C. et Bouguerra, M.L. (dir.). (1993). L'état de l'environnement dans le monde. Paris : La Découverte.

CMED - Commission mondiale sur l'environnement et le développement. (1988). Notre avenir à tous (Rapport Brundtland). Montréal : Éditions du Fleuve.

Cobast, E. (2005). L'insoutenable responsabilité des Hommes. Le regard des philosophes. In Gauchon, P. et Tellenne, C. (dir.), Géopolitique du développement durable. Rapport Antheios (p. 23-29). Paris : Presses Universitaires de France.

Delors, J. (1996). L'éducation, un trésor est caché dedans. Paris : Éditions Odile Jacob-UNESCO.

Ewald, F. (1992). L'expertise, une illusion nécessaire. In Theys, J. et Kalaora, B. (dir.), La Terre Outragée. Les experts sont formels ! (p. 203-210). Paris : Éditions Autrement.

Fourez, G. (1998). Se représenter et mettre en œuvre l'interdisciplinarité à l'école. Revue des sciences de l'éducation, 24(1), 31-50.

Jacquard, A. (1991). Voici le temps du monde fini. Paris : Éditions Seuil.

Jacquard, A. (2000). À toi qui n'es pas encore né(e). Paris : Calmann-Lévy.

Morin, E. (1990). Introduction à la pensée complexe. Paris : ESF Éditions.

Morin, E. (1992). Pour une pensée écologisée. In Theys, J. et Kalaora, B. (dir.), La Terre Outragée. Les experts sont formels! (p. 66-77). Paris : Éditions Autrement.

Morin, E. (1999). Les sept savoirs nécessaires à l'éducation du futur. Paris : UNESCO.

Morin, E. (2007). La démocratie cognitive et la réforme de la pensée. San Salvador : Instituto Internacional para el Pensamiento Complejo, Universidad del Salvador. Consulté en août 2009 sur : http://www.complejidad.org/cms/?q=node/15.

Ravetz, J. (1992). Connaissance utile, ignorance utile. In Theys, J. et Kalaora, B. (dir.), La Terre Outragée. Les experts sont formels! (p. 87-101). Paris : Éditions Autrement.

Theys, J. et Kalaora, B. (1992). Quand la science réinvente l'environnement. In Theys, J. et Kalaora, B. (dir.), La Terre Outragée. Les experts sont formels! (p. 15-49). Paris : Éditions Autrement.

\section{AUTEUR}

\section{MARYSE CLARY}

Maître de conférences honoraire, Maryse Clary a participé à des expérimentations ministérielles en matière d'éducation relative à l'environnement, tant en ce qui concerne la formation des enseignants qu'auprès des élèves. Elle a été responsable nationale de l'opération ENSI dans le cadre de l'OCDE-CERI et consultante en ErE pour le même organisme. Elle participe également à des recherches dans le cadre de l'Union Européenne. 